\title{
Guest Editorial Special Section on Integration of Electrochemical Energy Storage in Sustainable Energy Systems
}

Electrochemical energy storage, such as batteries and super-capacitors, is a rapidly advancing technology that promises to enable increased integration of renewable energy into the power grid. However, performance and cost challenges remain and it is often difficult to understand the role and value of energy storage in this context. Particular challenges include modelling and understanding degradation under various usage scenarios, optimal charge and discharge control, and robust, efficient monitoring and diagnostic approaches. The main objective of this Special Section in the IEEE Transactions on Sustainable Energy was to investigate exciting research challenges in design and control of electrochemical energy storage systems that are integrated into sustainable energy systems.

The Special Section contains 9 papers that were accepted from 12 papers submitted after selection based on extended abstracts. We were delighted and overwhelmed by the initial interest in this topic and received 101 extended abstracts, and the downselection process was challenging. The final accepted papers investigate a range of aspects of energy storage. At the scale of cells, two of the papers respectively investigate new approaches to state of charge estimation, and control of balancing circuits. At the scale of packs, where the majority of the papers focus, optimisation algorithms for sizing and control feature strongly. Two papers focus on the interaction between battery life and usage patterns, in providing multiple grid services, and in vehicle to grid applications respectively. Three papers focus on control of energy storage for dispatch of grid connected batteries incorporating photovoltaics in order to improve trading prospects and resilience. Finally, two papers focus on integration issues, specifically power electronics for grid energy storage, and analysis of hybrid energy storage systems incorporating batteries and supercapacitors.

I would like to recognize and thank deeply my coeditors for the large amounts of time they have put into this Special Section, reviewing abstracts, and assisting with the coordination of the review process of full papers. They are: Dr Federico Baronti, University of Pisa; Prof Chi-yung Chung, University of Saskatchewan; Prof Xiaosong $\mathrm{Hu}$, Chongqing University; Prof Michael Pecht, University of Maryland. I would also like to thank the Editor-in-Chief of the IEEE Transactions on Sustainable Energy, Prof Bikash Pal, for his help and guidance, and also Randi Scholnick for her support.

We hope that you find this special section engaging and useful, and that it becomes a key reference point for future work investigating energy storage integrated into the grid.

David A. Howey

Guest Editor-in-Chief

Department of Engineering Science

University of Oxford

Parks Road

Oxford OX1 3PJ

United Kingdom

email: david.howey@eng.ox.ac.uk 